\title{
Technical Advance
}

\section{Immunohistochemical Detection of Cell Growth Fraction in Formalin-Fixed and Paraffin-Embedded Murine Tissue}

\author{
Peter Birner, ${ }^{* \dagger}$ Marion Ritzi, ${ }^{\ddagger}$ Christine Musahl, ${ }^{\ddagger}$ \\ Rolf Knippers, ${ }^{\ddagger}$ Johannes Gerdes, ${ }^{\S}$ \\ Till Voigtländer, ${ }^{\dagger}$ Herbert Budka, ${ }^{\dagger}$ \\ Johannes A. Hainfellner ${ }^{\dagger}$ \\ From the Institutes of Clinical Pathology* and Neurology, ${ }^{\dagger}$ \\ University of Vienna, Vienna, Austria; the Department of \\ Biology, ${ }^{\ddagger}$ University of Konstanz, Konstanz, Germany; and the \\ Department of Immunology and Cell Biology, ${ }^{\S}$ Forschungsinstitut \\ Borstel, Borstel, Germany
}

Monoclonal antibody MIB-1 is a reliable tool for determining proliferating cells in human tissues, but does not react with the homologous mouse antigen and is therefore useless in experimental pathology using mice as model systems. Standard method for assessment of cellular proliferation in formalin-fixed, paraffin-embedded murine tissues is immunohistochemical detection of DNA synthesis using antibodies against exogenously injected 5-bromodeoxyuridine (BrdU), which is a tedious procedure and not useful for routine investigations. We tested monoclonal antibody MIB-5 and monoclonal and polyclonal anti-MCM3 antibodies as immunohistochemical proliferation markers for paraffin-embedded nonneoplastic and neoplastic tissues of wild-type and transgenic mice, compared to anti-BrdU immunostaining. Percentage of proliferating cells was determined with continuously decreasing antibody dilutions. Percentages of MIB-5 and anti-BrdU immunostained cells correlated strongly, as well as percentage of MIB-5-decorated cells and frequency of mitotic figures. Anti-MCM3 antibodies labeled significantly higher percentages of cells than anti-BrdU or MIB-5, and showed a linear decrease with increasing antibody dilutions. We conclude that MIB-5 detects reliably the cell growth fraction in formalin fixed, paraffin-embedded murine tissues, bypassing methodological drawbacks of BrdU. Anti-MCM3 antibodies are less useful for determination of proliferating cells although they might detect the fraction of cells remaining competent for proliferation. (Am J Patbol 2001, 158:1991-1996)
Uncontrolled cell proliferation is one of the main characteristics of tumors, ${ }^{1}$ and its rate predicts biological behavior and in many cases the clinical course of neoplasms. $^{2}$ The monoclonal antibody MIB-1 against the $\mathrm{Ki}-67$ antigen, which is expressed in the nuclei of all cells in $G_{1}, S$, and $G_{2}$ phases and mitosis, but not in the $G_{0}$ phase of the cell cycle, ${ }^{3,4}$ has become the gold standard for assessment of cell growth in paraffin-embedded specimens of human tissue. Such an easy-to-handle marker has not yet been established for murine tissues. Currently, immunohistochemical detection of exogenously injected 5-bromodeoxyuridine (BrdU) several hours before sacrifice is widely used for assessment of proliferation state in murine tissues. This nucleotide-analogue probe is integrated into the DNA of replicating cells during $S$ phase, ${ }^{5}$ and can be detected immunohistochemically in paraffin-embedded tissue sections afterward. However, this method has several shortcomings: first, BrdU is also integrated into cell DNA during DNA repair; thus, anti-BrdU stains cells engaged in DNA repair, in addition to proliferation. ${ }^{5}$ Second, the method requires an injecting of living animals, implying additional animal handling, and tissues untreated with BrdU cannot be analyzed. Third, repeated injections of BrdU before sacrifice of animals, as commonly performed in research laboratories, labels not only $S$ phase, but in addition all cells that passed the $S$ phase of cell cycle since the first injection, including mitotic figures and cells that have entered $G_{0}$ phase after division. Therefore, the fraction of anti-BrdU stained cells is variable, depending on duration and frequency of BrdU injections before sacrifice of animals. In recent years, the use of murine tumor models has steadily increased. ${ }^{6-8}$ Therefore, there is a growing need for a proliferation marker for routinely processed paraffin-embedded murine tissues, equivalent to MIB-1 for human tissues. Important requirements for an antibody useful as a tool for the determination of proliferating

Accepted for publication February 27, 2001.

Address reprint requests to Dr. Johannes A. Hainfellner, Institute of Neurology, AKH 4J, POB 48, Währinger Gürtel 18-20, A-1097 Vienna, Austria. E-mail: johannes.hainfellner@akh-wien.ac.at. 
cells are as follows: 1) the antibody should specifically identify proliferating cells; 2) the antibody should work in formalin-fixed, paraffin-embedded tissues without pretreatment of living animals; 3 ) the fraction of decorated cells should remain unchanged at different antibody dilutions instead of showing a gradient; and 4) the percentage and distribution of decorated cells should correlate closely with anti-BrdU staining results.

Recently, monoclonal antibody MIB-5 was suggested as a proliferation marker for murine tissues. ${ }^{9,10}$ MIB- 5 has been raised against bacterially expressed parts of the human $\mathrm{Ki}-67 \mathrm{cDNA},{ }^{10,11}$ and reacts with the Ki-67 protein in proliferating cells of several mammalian species. ${ }^{9,10}$ $\mathrm{Ki}-67$ protein is expressed as alternative splice products of $360 \mathrm{kd}$ and $320 \mathrm{kd}$. Both forms are bound to chromatin and seem to perform structural roles of unknown nature. ${ }^{12} \mathrm{Ki}-67$ protein is rapidly degraded once mammalian cells cease to proliferate, but although documented in numerous studies, the physiological meaning of this rapid Ki-67 degradation has yet to be established. ${ }^{12}$ However, the validity of MIB- 5 as a proliferation marker for murine tissues has not yet been thoroughly tested.

Antibodies against MCM3 have also been suggested as proliferation markers. ${ }^{13}$ The MCM family is highly conserved among eukaryotes and consists of at least six different nuclear proteins that are expressed in all eukaryotic cells where they perform essential functions in the regulation of chromatin replication. ${ }^{13,14}$ MCM3, a 105-kd protein, is expressed in proliferating cells where it is bound to chromatin before $S$ phase, but gradually released during $S$ phase and dispersed throughout the cell during mitosis. ${ }^{15}$ After growth arrest and differentiation, MCM3 disappears from cells with an estimated half life of 24 hours and is no longer synthesized in growth-arrested, differentiated cells. ${ }^{13}$ Anti-MCM3 antibodies stain cells in paraffin-embedded tissues of various species. ${ }^{3,13}$

In our study we tested the applicability of MIB-5 and anti-MCM3 antibodies as proliferation markers for paraffin-embedded nonneoplastic and neoplastic murine tissues as compared to anti-BrdU immunolabeling as the current gold standard.

\section{Materials and Methods}

\section{Animals}

We analyzed nonneoplastic tissues sampled from four wild-type C57BL/6 mice (two males, two females). Wildtype tissues included brain, gut, heart, thymus, lungs, kidney, spleen, liver, ovary, testes, skin, and skull and spinal column with bone marrow. We further analyzed 15 pituitary adenomas in RB-1 hemizygous mice carrying in addition the GFAP-V-src transgene (RB- $1^{+/-}$GFAP-V-src mice), 7 astrocytomas in TP53 hemizygous mice carrying in addition the GFAP-V-src transgene (TP53 ${ }^{+/-}$GFAP-Vsrc mice) or RB-1 ${ }^{+/-}$GFAP-v-src mice, and 16 GFAPexpressing nonglial tumors of soft tissue and bone and 1 lymphoma of the spleen in $15 \mathrm{TP}^{+1-}$ GFAP-v-src mice. ${ }^{16}$ The gut of one male white laboratory rat was also studied. All mice were injected 16 and 4 hours before sacrifice intraperitoneally with $0.2 \mathrm{ml}$ of a $5 \mathrm{mg} / \mathrm{ml} \mathrm{BrdU}$ solution. Mice were killed by cervical dislocation. After removal, tissues were fixed in formalin for 24 hours, and embedded in paraffin. Skull and spinal column were decalcified in $0.5 \mathrm{~mol} / \mathrm{L}$ of ethylenediaminetetraacetic acid, $\mathrm{pH} 8.0$, for 10 days, before paraffin-embedding. For comparison of slowly and fast proliferating tissues, paraffin blocks were prepared that contained both brain (harbors postmitotic nerve cells) and gut (harbors briskly proliferating epithelium). Institutional review board approval for this study was obtained.

\section{Immunohistochemistry}

After removal of paraffin with xylene, endogenous peroxidase was blocked in all tissues with $3 \% \mathrm{H}_{2} \mathrm{O}_{2}$ in methanol. All tissue sections were pretreated in a microwave oven in citrate buffer at $\mathrm{pH} 6.0$ for 10 minutes at $100^{\circ} \mathrm{C}$ at 300 W. Unspecific binding sites were blocked using washing buffer 1 (no. K5006; DAKO, Glostrup, Denmark) before incubation with the primary antibody. Slides were incubated for 1 hour at room temperature with the primary antibodies. As primary antibodies, we used monoclonal antibody MIB-5 (antibody concentration $0.2 \mathrm{mg} / \mathrm{ml}$, no. 2093; Immunotech, Marseille, France ) (this antibody is now available from DAKO, Glostrup, Denmark), a biotinylated monoclonal anti-BrdU antibody (no. MD 5215; Caltag Laboratories, Burlingame, CA) (antibody concentration $0.2 \mathrm{mg} / \mathrm{ml}$ ), a monoclonal anti-MCM3 antibody (antibody concentration $2.5 \mathrm{mg} / \mathrm{ml}$ ), and a polyclonal anti-MCM3 antibody (antibody concentration $0.2 \mathrm{mg} / \mathrm{ml}$ ). Preparation of the polyclonal anti-MCM3 antibody has been described previously. ${ }^{17}$ Monoclonal anti-MCM3 was prepared using a standard protocol against bacterially expressed human $\mathrm{MCM} 3$ protein (data to be published). To avoid background because of secondary antibody binding to mouse immunoglobulins in tissue, MIB-5 was biotinylated using the Animal Research Kit Peroxidase (no. K3954; DAKO) according to the manufacturers instructions before immunostaining. As a detection system, a streptavidin-peroxidase complex included in the kit was used. After microwave treatment, specimens were digested for 5 minutes with $0.03 \%$ proteinase K (no. P-6556; Sigma-Aldrich, St. Louis, MO). Detection of anti-BrdU binding was performed using an avidinperoxidase system (no. A-3151; Sigma-Aldrich). To minimize background staining of the monoclonal anti-MCM3 antibody, the Mouse on Mouse (M.O.M.) kit (no. PK-2200; Vector, Burlingame, CA) was used as a detection system. For detection of polyclonal anti-MCM3 binding, an antirabbit secondary antibody (no. Z0196; DAKO) and a peroxidase-anti-peroxidase system (no. Z0113; DAKO) were used. Diaminobenzidine was used as chromogen for all immunostainings, counterstaining was performed with hemalaun. Omission or substitution of MIB-5 or monoclonal anti-MCM3 antibody by isotype-matched nonspecific immunoglobulins was used as negative controls. Binding specificity of polyclonal MCM3 antibody was controlled by replacing the antibody with nonspecific rabbit serum. 
A
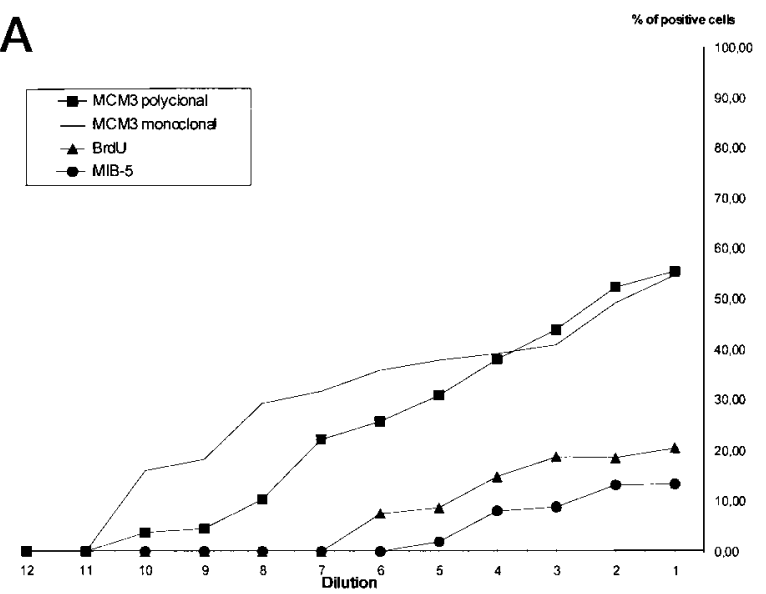

\begin{tabular}{cl|c|c|c|c}
\multicolumn{5}{c|}{ Dilution } & \multicolumn{2}{|c|}{ Antibody } & BrdU & MIB-5 & MCM3 polyclonal & MCM3 monoclonal \\
\hline $\mathbf{1}$ & $\mathbf{1 : 1 0}$ & $20.48 \pm 4.45$ & $13.36+2.31$ & $55.2 \pm 4.43$ & $54.72 \pm 7.41$ \\
$\mathbf{2}$ & $\mathbf{1 : 2 0}$ & $18.52 \pm 6.62$ & $13.21+2.54$ & $52.45 \pm 9.41$ & $49.18 \pm 4.92$ \\
$\mathbf{3}$ & $\mathbf{1 : 5 0}$ & $18.73 \pm 4.89$ & $8.85+2.95$ & $43.84 \pm 6.16$ & $40.85 \pm 6.79$ \\
$\mathbf{4}$ & $\mathbf{1 : 1 0 0}$ & $14.82 \pm 5.79$ & $8.14+1.63$ & $37.91 \pm 8.18$ & $39.10 \pm 2.92$ \\
$\mathbf{5}$ & $\mathbf{1 : 2 0 0}$ & $8.77 \pm 3.90$ & $1.90+1.79$ & $30.91 \pm 12.44$ & $37.67 \pm 5.29$ \\
$\mathbf{6}$ & $\mathbf{1 : 4 0 0}$ & $7.54 \pm 3.54$ & 0 & $25.77 \pm 10.82$ & $35.81 \pm 3.62$ \\
7 & $\mathbf{1 : 8 0 0}$ & 0 & 0 & $22.17 \pm 7.66$ & $31.70 \pm 6.39$ \\
$\mathbf{8}$ & $\mathbf{1 : 1 5 0 0}$ & 0 & 0 & $10.19 \pm 1.98$ & $29.24 \pm 5.62$ \\
9 & $\mathbf{1 : 3 0 0 0}$ & 0 & 0 & $4.49 \pm 3.27$ & $18.22 \pm 2.11$ \\
$\mathbf{1 0}$ & $\mathbf{1 : 4 0 0 0}$ & 0 & 0 & $3.72 \pm 4.24$ & $15.93 \pm 2.55$ \\
$\mathbf{1 1}$ & $\mathbf{1 : 8 0 0 0}$ & 0 & 0 & 0 & 0 \\
$\mathbf{1 2}$ & $\mathbf{1 : 1 6 0 0 0}$ & 0 & 0 & 0 & 0
\end{tabular}

\section{B}

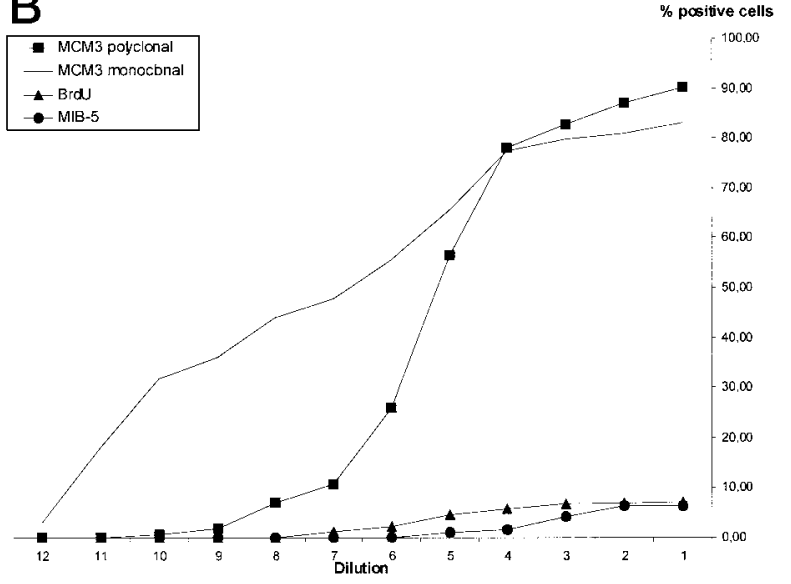

\begin{tabular}{|c|c|c|c|c|c|}
\hline & & \multicolumn{4}{|l|}{ Antibody } \\
\hline \multicolumn{2}{|c|}{ Dilution } & BrdU & MIB-5 & MCM3 polyclonal & MCM3 monoclonal \\
\hline $\mathbf{1}$ & $1: 10$ & $7.11 \pm 1.92$ & $6.28 \pm 2.25$ & $90.19 \pm 1.45$ & $82.93 \pm 5.68$ \\
\hline 2 & $1: 20$ & $6.99 \pm 1.29$ & $6.23 \pm 2.21$ & $86.89 \pm 1.50$ & $80.80 \pm 7.21$ \\
\hline 3 & $1: 50$ & $6.66 \pm 0.86$ & $4.09 \pm 0.59$ & $82.68 \pm 4.89$ & $79.72 \pm 6.52$ \\
\hline 4 & $1: 100$ & $5.68 \pm 1.35$ & $1.53 \pm 1.03$ & $77.87 \pm 1.04$ & $77.22 \pm 10.95$ \\
\hline 5 & $1: 200$ & $4.56 \pm 1.62$ & $1.03 \pm 0.68$ & $56.24 \pm 9.12$ & $65.49 \pm 10.57$ \\
\hline 6 & $1: 400$ & $2.21 \pm 0.85$ & 0 & $25.84 \pm 8.50$ & $55.52 \pm 7.82$ \\
\hline 7 & $1: 800$ & $1.11 \pm 1.06$ & 0 & $10.61 \pm 1.05$ & $47.63 \pm 12.08$ \\
\hline 8 & $1: 1500$ & 0 & 0 & $7.00 \pm 1.61$ & $43.88 \pm 3.47$ \\
\hline 9 & $1: 3000$ & 0 & 0 & $1.82 \pm 1.60$ & $35.95 \pm 2.75$ \\
\hline 10 & $1: 4000$ & 0 & 0 & $0.68 \pm 0.42$ & $31.64 \pm 2.63$ \\
\hline 11 & $1: 8000$ & 0 & 0 & 0 & $18.02 \pm 15.70$ \\
\hline 12 & $1: 16000$ & 0 & 0 & 0 & $3.04 \pm 3.63$ \\
\hline
\end{tabular}

Figure 1. A: Percentages of immunostained epithelial cells in small intestine (mean value \pm SD of positive cells determined as described in Materials and Methods). B: Percentages of immunostained tumor cells in pituitary adenoma (mean value \pm SD of positive cells determined as described in Materials and Methods).

\section{Analysis}

In a first step, the percentages of immunostained cells were determined in samples of small intestine of two wild-type mice and in pituitary adenomas of two RB- $1^{+/-}$ GFAP-V-src mice. Immunostaining was performed with anti-BrdU, MIB-5, and monoclonal/polyclonal anti-MCM3 as primary antibodies on subsequent serial sections. In the small intestine, the fraction of immunostained epithelial cells was determined on cross-cut villi showing the epithelium as monolayer. Epithelial cells were counted from the lowest point of the crypt to the tip of the villus and the percentage of positive cells was calculated. The mean value of the percentages of decorated cells in three villi was used for further calculations. In pituitary adenomas, the fraction of immunostained cells was determined using an eyeshield and counting tumor cells at a magnification of $\times 400$ in three subsequent microscopic fields in the tissue area showing the highest density of immunostained cells. The percentage of decorated cells was calculated for each field and the mean value of the three fields was used for further calculations. Immunostaining and assessment of decorated cells was performed for all primary antibodies at dilutions of 1:10, 1:20, 1:50, 1:100, $1: 200,1: 400,1: 800,1: 1500,1: 3000,1: 4000,1: 8000$, and 1:16,000 (Figure 1, A and B). In addition to conventional light microscopic examination, specimens were also investigated by computerized image analysis using the Advanced Cellular Imaging System (ACIS; Chromavision, San Juan Capistrano, CA) with the Ki-67 module according to the manufacturer's instructions. For assessment of significant differences of cells stained by anti-BrdU, MIB-5, or monoclonal/polyclonal anti-MCM3 in small intestine or pituitary adenomas we used the paired Friedman test with antibody dilution as pairing factor. Significant results were further investigated by the sign tests with adjustment according to Bonferroni for multiple testing. A $P$ value $\leq 5 \%$ was considered as significant.

MIB-5 immunostaining was then performed at a dilution of 1:20 in a series of murine tumors comprising 15 pituitary adenomas, 7 astrocytomas, 16 tumors of soft tissue and bone, and 1 lymphoma (Animals section). The MIB-5 labeling index was determined as described above. In addition, mitotic frequency was determined counting mitotic figures in 10 high-power fields (HPF) at a magnification of $\times 400$. The correlation of MIB-5-labeling index and mitotic frequency was investigated by Pearson's coefficient of correlation.

\section{Results}

All antibodies showed distinct nuclear staining of proliferating cells of epithelia, internal organs, and bone marrow, and of tumor cells. In various epithelia (skin, bladder, airway) MIB-5 binding was restricted to nuclei of basal cells, thus showing identical patterns as reported for MIB-1 in corresponding human tissues. ${ }^{10,18}$ Antibodies against MCM3 intensely stained nuclei in the basal epidermal layer, but in addition also nuclei of cells in the upper epidermal layers although with diminishing inten- 

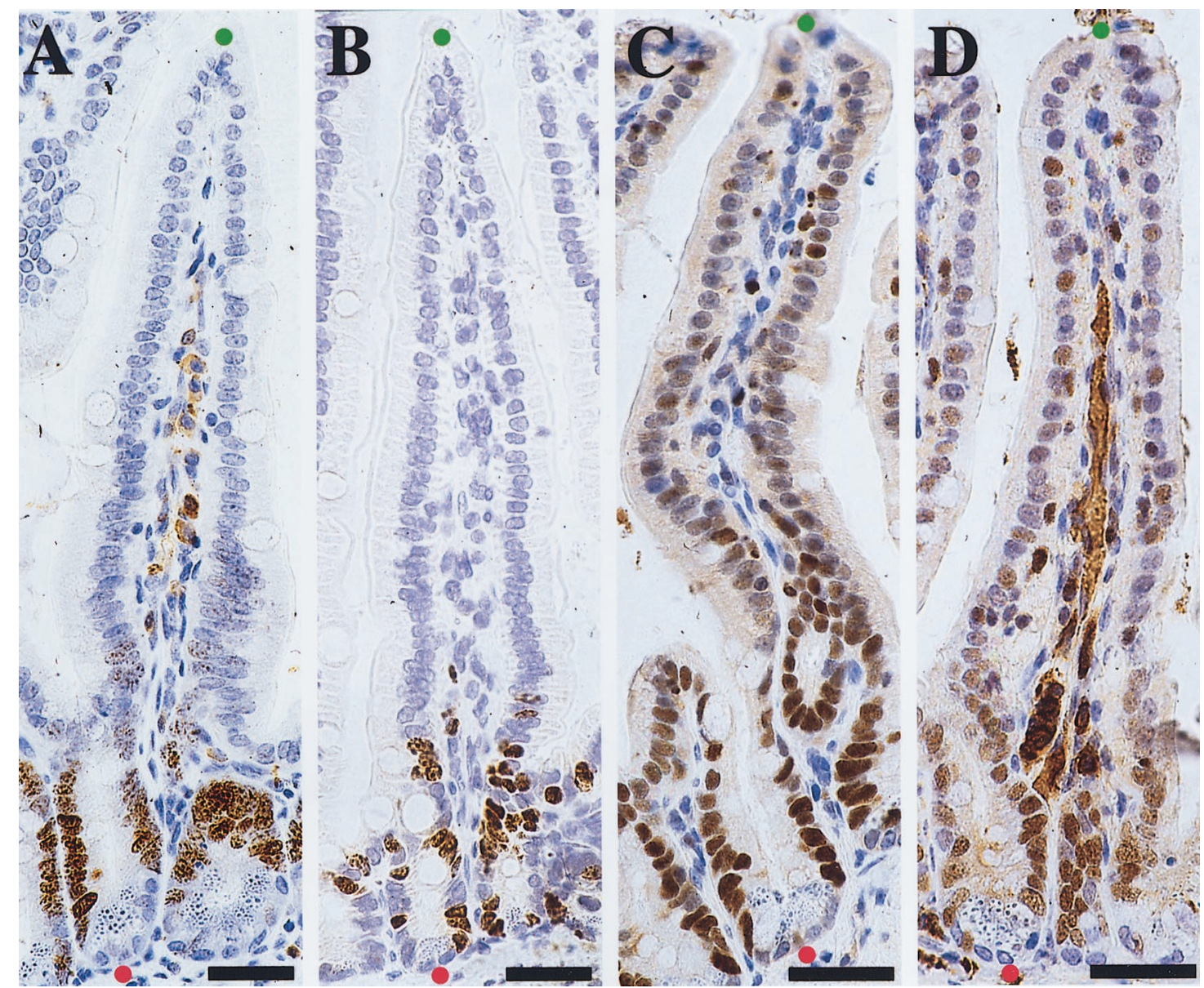

Figure 2. Representative results of MIB-5, anti-BrdU, and anti-MCM3 immunostaining on small intestine (immunoperoxidase). The red dots indicate the lowest points of the crypts and the green dots indicate the tips of villi in the small intestine. The percentage of immunostained cells was determined in the epithelial stretches between the red and green dots. Scale bar, $30 \mu \mathrm{m}$. A: MIB-5, 1:20. B: BrdU, 1:20. C: MCM3 polyclonal, 1:100. D: MCM3 monoclonal, 1:50.

sities, as already observed in a previous study. ${ }^{13}$ Immunostaining of the esophageal and respiratory epithelium with anti-MCM3 antibodies showed staining patterns similar to the skin, although the reaction was more confined to the basal cell layers. Nuclear staining faded at higher dilutions (Figure 1, a and b). Even at low antibody dilutions, there was no staining of neuronal nuclei with any of the antibodies. Using a commercially available biotinylation kit that includes a streptavidin-peroxidase complex system for detection of primary antibody binding, we achieved a clean and distinct immunostaining signal with MIB-5 equivalent to MIB-1 staining of human tissues.

Computerized image analysis of MIB-5-stained slides yielded similar values as compared to conventional light microscopic investigation (data not shown), whereas cytoplasmic background of some of the anti-BrdU and antiMCM3 immunostained sections did not allow computerized image analysis. Therefore, quantitative assessment of immunostained cells was performed by means of conventional light microscopic analysis. Figure 1, a and b, shows compiled data of quantitative assessment and illustrates for all antibodies the percentages of decorated cells depending on antibody dilution. Figures 2 and 3 show samples of immunostaining.
Analyzing the percentages of positive cells statistically, the Friedman test revealed significant differences between MIB-5, anti-BrdU, and anti-MCM3 antibodies both in the small intestine and pituitary adenoma $(P<$ 0.001 , Friedman test). We scrutinized the differences by subsequent pairwise testing using sign tests with Bonferroni adjustment and found significant differences $(P<$ 0.02 ) between MIB-5 and the two anti-MCM3-antibodies used, as well as between anti-BrdU and both anti-MCM3 antibodies, both in the small intestine as well as in pituitary adenoma. We did not observe significant differences between MIB-5 and anti-BrdU, and between monoclonal and polyclonal anti-MCM3. Repeated immunostaining with MIB-5 on small intestine specimens yielded highly reproducible results. The percentage of anti-BrdU-immunostained cells in the small intestine was slightly higher than that of MIB-5-immunostained cells (Figure 1, a and b); this difference was, however, not significant after adjustment for multiple testing.

Analyzing a series of tumors, MIB-5-decorated cells ranged from 5.8 to $47.2 \%$ in astrocytomas $(n=7)$, from 12.1 to $78.1 \%$ in tumors of soft tissue and bone $(n=16)$, and from 0.3 to $8.8 \%$ in pituitary adenomas $(n=15)$. [Labeling of up to $8.8 \%$ of the murine pituitary adenoma 


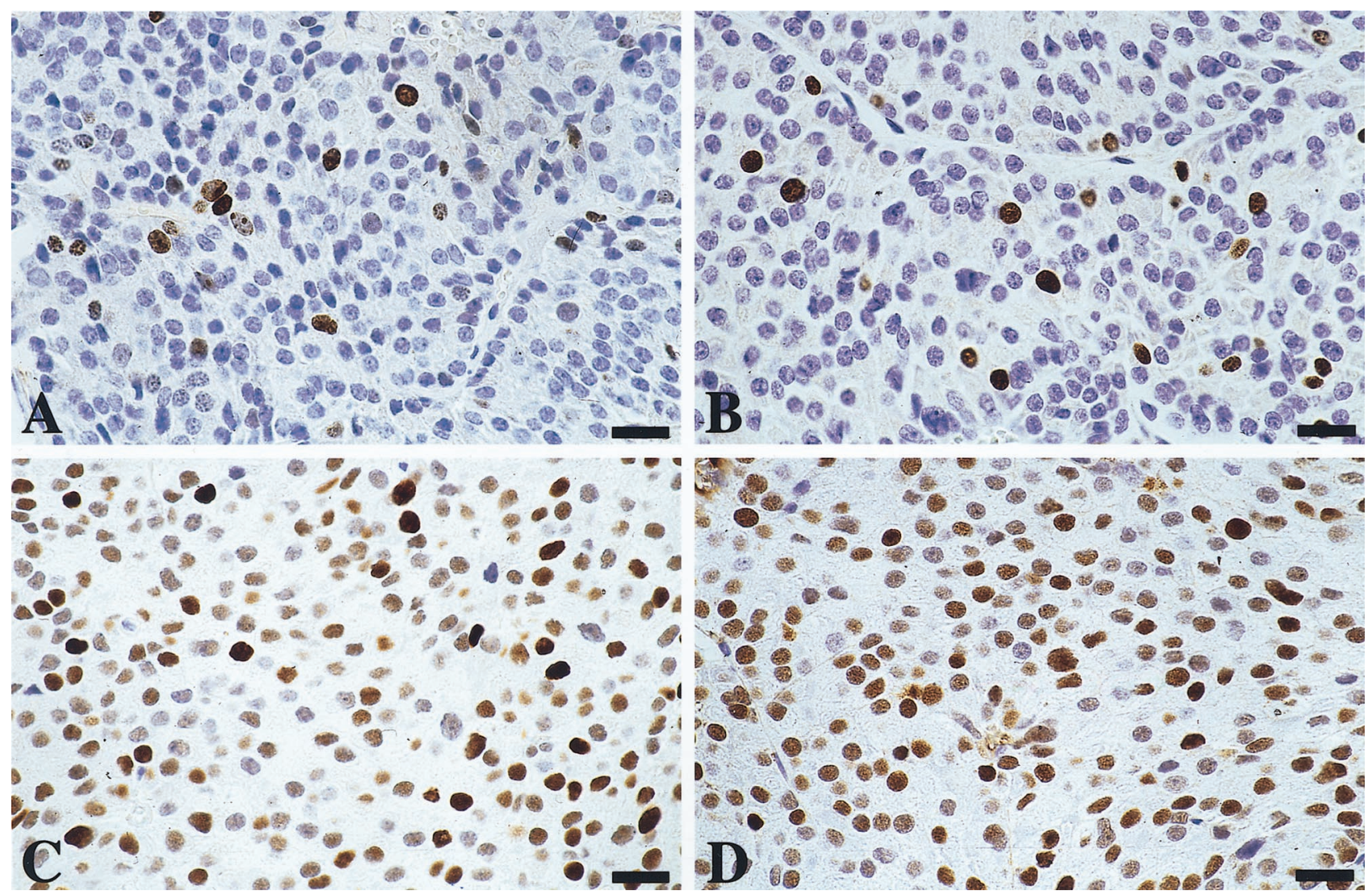

Figure 3. Representative results of MIB-5, anti-BrdU, and anti-MCM3 immunostaining on pituitary adenoma (immunoperoxidase). Scale bar, $20 \mu \mathrm{m}$. A: MIB-5, 1:20. B: BrdU, 1:20. C: MCM3 polyclonal, 1:100. D: MCM3 monoclonal, 1:50.

cells is rather high as compared to human pituitary adenomas, in which MIB-1 labeling has been reported to be $<5 \% .{ }^{19}$ Interspecies differences and differences of the genetic basis of human pituitary adenomas and our murine pituitary adenomas are possible explanations for the slight difference of MIB-1 and MIB-5 labeling.] In the case of lymphoma, $29.4 \%$ of cells showed MIB-5 binding. The number of mitotic figures/10 HPF ranged from 4 to 35 in astrocytomas, from 12 to 55 in tumors of soft tissue and bone, and from 0 to 13 in pituitary adenomas. The case of lymphoma showed 22 mitotic figures/10 HPF. There was a strong correlation between the percentage of MIB-5decorated cells and number of mitotic figures/10 HPF ( $r=0.93, P<0.001$, Pearson's coefficient of correlation) (Figure 4).

In rat gut, MIB-5 immunostaining showed a similar pattern as in mouse gut with prominent labeling of epithelial cells in the crypt and negative epithelial cells on the villous surfaces.

\section{Discussion}

Immunohistochemical detection of exogenously injected BrdU is currently the only available reliable tool for assessment of the S-phase fraction of cells in murine tissues. Anti-BrdU immunostaining was introduced in 1982 as a safe alternative to $\left[{ }^{3} \mathrm{H}\right]$-thymidine microautoradiography. ${ }^{20}$ Although anti-BrdU immunostaining has proven as an important method for detection of cell proliferation, there are some methodological drawbacks (as detailed in the Introduction section). Because of the methodological drawbacks, results based on anti-BrdU staining and data interpretation are sometimes ambiguous. ${ }^{21}$ In our study, we injected mice 16 and 4 hours before sacrifice according to a protocol commonly used in research laboratories. Using this protocol, we BrdU-labeled all cells that passed S phase during the 16 hours before sacrifice of the animals. The percentage of BrdU-labeled cells was equal or even slightly higher, as compared to MIB-5 labeling of the Ki-67 antigen.

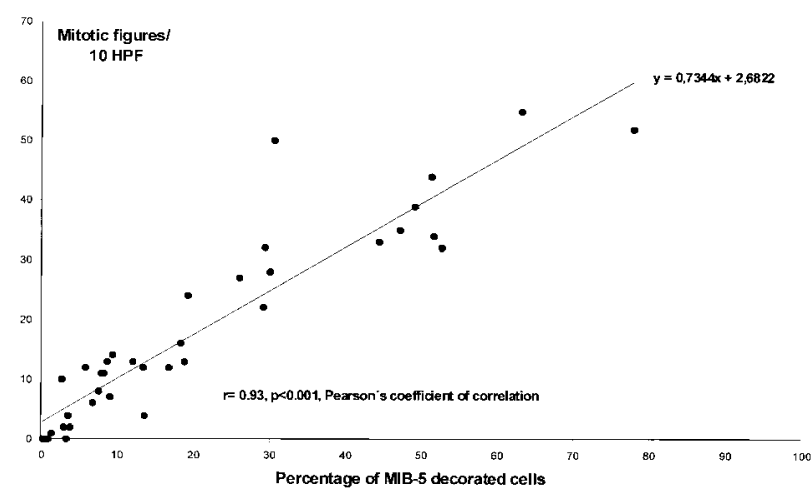

Figure 4. Correlation between the percentage of MIB-5 decorated cells and number of mitotic figures/10 HPF in a series of murine tumors $(n=39$, for more detailed information on the tumors see Materials and Methods). 
In our study, we tested systematically the validity of MIB-5 and anti-MCM3 antibodies as cell proliferation markers on routinely processed, paraffin-embedded murine tissues as an alternative to anti-BrdU staining. All of the antibodies labeled nuclei of proliferating cells of various tissues and of tumor cells, but no neuronal nuclei. At different dilutions MIB-5 labeled similar cell fractions only in proliferating tissue zones and the results closely correlated with anti-BrdU staining. MIB-5 proved thus as a reliable and reproducible tool to detect the fraction of proliferating cells. In contrast, anti-MCM3 antibodies labeled significantly higher percentages of cells as compared to anti-BrdU and MIB-5 staining. In the small intestine, anti-MCM3 stained some of the postmitotic epithelial cells on the villous surface, in addition to proliferating epithelial cells in the crypt (Figure 2). With increasing antibody dilutions, the percentage of anti-MCM3-immunostained cells showed a linear decrease. Anti-MCM3 immunostaining seems to parallel the situation in assessment of proliferation rate using immunostaining for proliferating cell nuclear antigen, where the number of decorated cells depends strongly on the sensitivity of the immunocytochemical system used, ${ }^{22}$ thus causing little reproducibility of staining results after repeated immunostaining or when using different staining protocols. The presence of MCM3 in a cell fraction that is larger than the fraction containing either $\mathrm{BrdU}$ or Ki-67 may be explained by a relatively long half-life of MCM3 protein. MCM3 protein has been shown to disappear more slowly after initiation of differentiation than the Ki-67 antigen. ${ }^{13}$ Thus, MCM3 protein may still be detectable for some time in early $G_{0}$ phase, after the cell has accomplished its cycle. However, even at low dilutions, anti-MCM3 antibodies did not stain neuronal cell nuclei in the brain.

In conclusion, our results show that anti-MCM3 antibodies are not useful for quantitative assessment of proliferating cells, although they might be useful for detecting the fraction of cells remaining competent for proliferation. ${ }^{23}$ In contrast, monoclonal antibody MIB-5 shows staining results in murine tissues, equivalent to MIB-1 staining of human tissues. Assessment of cell proliferation with the MIB5 antibody allows bypassing the methodological drawbacks of anti-BrdU staining. Thus, we propose antibody MIB-5 as the first-choice cell proliferation marker for routinely processed formalin-fixed and paraffin-embedded murine tissues.

\section{Acknowledgments}

We thank Birgit Schmid and Helga Flicker for excellent technical assistance.

\section{References}

1. Tubiana M: Tumor cell proliferation kinetics and tumor growth rate. Acta Oncol 1989, 28:113-121

2. Tubiana M, Courdi A: Cell proliferation kinetics in human solid tumors: relation to probability of metastatic dissemination and long-term survival. Radiother Oncol 1989, 15:1-18

3. Gerdes J, Schwab U, Lembke H, Stein H: Production of a mouse monoclonal antibody reactive with a human nuclear antigen associated with cell proliferation. Int J Cancer 1983, 31:13-20

4. Gerdes J, Li L, Schlüter C, Duchrow M, Wohlenberg C, Gerlach C, Stahmer I, Kloth S, Brandt E, Flad H: Immunobiochemical and molecular biologic characterization of the cell proliferation associated nuclear antigen that is defined by monoclonal antibody Ki-67. Am J Pathol 1991, 138:867-873

5. Selden JR, Dolbeare F, Clair JH, Nichols WW, Miller JE, Kleemeyer KM, Hyland RJ, DeLuca JG: Statistical confirmation that immunofluorescent detection of DNA repair in human fibroblasts by measurement of bromodeoxyuridine incorporation is stoichiometric and sensitive. Cytometry 1993, 14:154-167

6. Lovejoy EA, Clarke AR, Harrison DJ: Animal models and the molecular pathology of cancer. J Pathol 1997, 181:130-135

7. Hotz HG, Hines OJ, Foitzik T, Reber HA: Animal models of exocrine pancreatic cancer. Int J Colorect Dis 2000, 15:136-143

8. Leibovici J, Wolman M: Animal models for tumor progression. Anticancer Res 1984, 4:165-168

9. Ito T, Mitui H, Udaka N, Hayashi H, Okudela K, Kanisawa M, Kitamura $\mathrm{H}$ : Ki-67 (MIB 5) immunostaining of mouse lung tumors induced by 4-nitroquinoline 1-oxide. Histochem Cell Biol 1998, 110:589-593

10. Gerlach C, Golding M, Larue L, Alison MR, Gerdes J: Ki-67 immunoexpression is a robust marker of proliferative cells in the rat. Lab Invest 1997, 77:697-698

11. Schlüter $\mathrm{C}$, Duchrow M, Wohlenberg $\mathrm{C}$, Becker MH, Key G, Flad H, Gerdes J: The cell proliferation-associated antigen of antibody Ki-67: a very large, ubiquitous nuclear protein with numerous repeated elements, representing a new kind of cell cycle-maintaining proteins. J Cell Biol 1993, 123:513-522

12. Scholzen T, Gerdes J: The Ki-67 protein: from the known and the unknown. J Cell Physiol 2000, 182:311-322

13. Musahl C, Holthoff HP, Lesch R, Knippers R: Stability of the replicative $\mathrm{Mcm} 3$ protein in proliferating and differentiating human cells. Exp Cell Res 1998, 241:260-264

14. Madine MA, Khoo C, Mills AD, Laskey RA: MCM3 complex required for cell cycle regulation of DNA replication in vertebrate cells. Nature 1995, 375:421-424

15. Krude T, Musahl C, Laskey RA, Knippers R: Human replication proteins hCdc21, hCdc46 and P1Mcm3 bind chromatin uniformly before S-phase and are displaced locally during DNA replication. J Cell Sci 1996, 109:309-318

16. Maddalena AS, Hainfellner JA, Hegi ME, Glatzel M, Aguzzi A: No complementation between TP53 or RB-1 and v-src in astrocytomas of GFAP-v-src transgenic mice. Brain Pathol 1999, 9:627-637

17. Burkhart R, Schulte D, Hu D, Musahl C, Gohring F, Knippers R: Interactions of human proteins $\mathrm{P} 1 \mathrm{Mcm} 3$ and P1Cdc46. Eur J Biochem 1995, 228:431-438

18. Gerdes J, Becker MH, Key G: Immunohistological detection of tumor growth fraction (Ki-67 antigen) in formalin-fixed and routinely processed tissues. J Pathol 1992, 168:85-87

19. Abe T, Sanno N, Osamura YR, Matsumoto K: Proliferative potential in pituitary adenomas: measurement by monoclonal antibody MIB-1. Acta Neurochir 1997, 139:613-618

20. Gratzner HG: Monoclonal antibody to 5-bromo- and 5-iododeoxyuridine: a new reagent for detection of DNA replication. Science 1982, 218:474-476

21. Gould E, Gross CG: New neurons: extraordinary evidence or extraordinary conclusion? Science 2000, 288:771

22. Khoshyomn S, Maier H, Morimura T, Kitz K, Budka H: Immunostaining for proliferating cell nuclear antigen: its role in determination of proliferation in routinely processed human brain tumor specimens. Acta Neuropathol 1993, 86:582-589

23. Williams GH, Romanowski P, Morris L, Madine M, Mills AD, Stoeber K, Marr J, Laskey RA, Coleman N: Improved cervical smear assessment using antibodies against proteins that regulate DNA replication. Proc Natl Acad Sci USA 1998, 95:14932-14937 